\section{MS17-O2 Structural studies of M-type ferrites used as template layers for the growth of oriented Y-type ferrites through chemical solution deposition method \\ Radomir Kuzel ${ }^{1}$, Josef Bursik ${ }^{2}$, Dorota Pulmannova², Robert Uhrecky $^{2}$, Milan Dopita ${ }^{1}$}

1. Charles University in Prague, Faculty of Mathematics and Physics, Ke Karlovu 5, Praha 2, Czech Republic

2. Institute of Inorganic Chemistry, Czech Academy of Sciences, Rez near Prague, Czech Republic

email: kuzel@karlov.mff.cuni.cz

Thin films of trigonal $\mathrm{Ba}_{2} \mathrm{Zn}_{2} \mathrm{Fe}_{12} \mathrm{O}_{22}$ (Y-type) ferrite were prepared by the chemical solution deposition using $\mathrm{SrTiO}_{3}(111)$ substrates covered with hexagonal magnetoplumbite (M-Type) seed layers. Seven M phases with different chemical composition, magnetic character and lattice misfit $(-0.8 \%$ to $-7.0 \%)$ values were investigated in their use as template and buffer layers. The films were studied by X-ray diffraction, atomic force microscopy and electron back-scatter diffraction. X-ray diffraction analysis in parallel beam setup with Eulerian cradle was concentrated on the precise determination of lattice parameters, studies of preferred orientation by symmetric and asymmetric scans ( $w$ and $\mathrm{j}$ ) and studies of possible stresses for all the layers by measurement of reflections at different inclinations $\mathrm{j}$ and $\mathrm{y}$. For this, correct reference values of lattice parameters are required in particular for non-cubic phases. For most of the phases tested, the values are either completely missing in PDF4+ database or often a number of values can be found there for a single phase. Therefore some data analysis of PDF4+ with respect to the statistics and/or also to the stoichiometry was performed.

For all the seed layers strong out-of-plane (0001) orientation of M- and Y-films was observed but with somewhat different degree. The in-plane orientation depended on misfit between the M-interlayer and substrate and also M-interlayer and the top Y-film. The optimum growth was reached using $\mathrm{SrTiO}_{3}(111) /(\mathrm{BaSr})(\mathrm{GaAl}){ }_{12} \mathrm{O}_{19}$, $\mathrm{SrTiO}_{3}(111) / \mathrm{Ba}(\mathrm{FeAl}){ }_{12} \mathrm{O}_{19}$, and $\mathrm{SrTiO}_{3}(111) / \mathrm{SrGa}_{12} \mathrm{O}_{10}$ substrate/seed layer architectures. In general, the best results were achieved when the misfit values between the seed layer and substrate, and between the seed layer and top Y-layer are approximately equal and when the surface of seed layers are formed by hexagons. Then single domain perfect hexagon-on-hexagon orientation was observed for M-film. For Y-layer also the in-plane orientation $(001)_{\mathrm{Y}} / /(111)_{\mathrm{STO}} / /$ and $[100]_{\mathrm{Y}} / /$ sTO was observed but six maxima detected in $\mathrm{j}$-scans instead of three confirmed in-plane obverse/reverse twinning. The results demonstrated the possibilities of the intelligent material design (namely anisotropy manipulation) for this important class of magnetic materials by proper design of key constituents: lattice misfits and surface topography of seeding structures.

Keywords: powder diffraction, oriented thin films, preferred orientation

\section{MS17-03 Pore Shape Determination in Mesoporous Ordered Silica Films}

Gerhard Popovski ${ }^{1}$, Roland Morak ${ }^{1}$, Parvin Sharifi ${ }^{2}$, Heinz Amenitsch $^{3}$, Oskar Paris ${ }^{1}$

1. Montanuniversitaet Leoben

2. Max-Planck-Institut fuer Kohlenforschung,

3. Graz University of Technology

email: gerhard.popovski@unileoben.ac.at

Mesoporous materials are often templated by organic materials that give a scaffold for precursor molecules. The organic material is removed by a calcination step, which leads to shrinkage of the whole structure. In the case of thin films the shrinkage is largely inhibited in two dimensions leading to a uniaxial deformation of pore lattice and of the pores themselves.

Gracing incidence small angle $\mathrm{x}$-ray scattering (GISAXS) of ordered mesoporous films show well-defined Bragg peaks. The deformation of the lattice can be determined from the positions of the Bragg-peaks after correction for reflection and refraction effects at the film interfaces. The intensities of the peaks can be corrected for geometric effects, i.e. the rotational symmetry around the surface normal and the curvature of the Ewald-sphere intersecting the peaks at different positions in reciprocal space. It is then possible to approximate the corrected peak intensities by a model for elliptically shaped cross sections of the pores. The pores are found to by deformed similar to the lattice. Pores and lattice are slightly more deformed in spin coated films than in dip-coated ones.

Keywords: mesoporous films, GISAXS 